\title{
Hybrid Modelling in Biology: a Classification Review
}

\author{
A. Stéphanou ${ }^{1 *}$, V. Volpert ${ }^{2,3,4}$ \\ ${ }^{1}$ UJF-Grenoble 1, CNRS, Laboratory TIMC-IMAG/DyCTiM, UMR 5525, 38041 Grenoble, France \\ ${ }^{2}$ Institut Camille Jordan, UMR 5208 CNRS, University Lyon 1, 69622 Villeurbanne, France \\ ${ }^{3}$ INRIA Team Dracula, INRIA Lyon La Doua, 69603 Villeurbanne, France \\ ${ }^{4}$ Institute of Numerical Mathematics, Russian Academy of Sciences, 119333 Moscow, Russia
}

\begin{abstract}
This paper presents a general review on hybrid modelling which is about to become ubiquitous in biological and medical modelling. Hybrid modelling is classically defined as the coupling of a continuous approach with a discrete one, in order to model a complex phenomenon that cannot be described in a standard homogeneous way mainly due to its inherent multiscale nature. In fact, hybrid modelling can be more than that since any types of coupled formalisms qualify as being hybrid. This review first presents the evolution and current context of this modelling approach. It then proposes a classification of the models through three different types that relate to the nature and level of coupling of the formalisms used.
\end{abstract}

Keywords and phrases: complex system, discrete-continuous, hybrid model, multiscale, system biology.

Mathematics Subject Classification: 35Q92, 68R0, 92B05, 92C42, 92C50, 93A30, $93 \mathrm{C} 55$

\section{Introduction}

The term hybrid refers to something of a mixed origin or composition. Hybrid modelling in biology is mainly understood as the coupling of continuous and discrete formulations. Classically reaction-diffusion systems of partial differential equations, representing the evolutions of chemical concentrations or densities, are coupled with cellular automata or agent based models. The latter are used to represent the individual behaviour of some entities of interest on which we wish to assign individual characteristics. The state and/or characteristics of these entities evolve with the external continuous chemical (or other) fields. Although most of the models in biomedicine follow this pattern, hybrid modelling can be much more than that. In its most general definition, a hybrid model corresponds to any interaction or coupling between two or more models that are not based on the same formalism. For example deterministic and stochastic, global and local, phenomenological and physically based, etc.

Many special issues in journals $[27,34]$ or reviews have been written on the topic of hybrid modelling in biology, either explicitly or implicitly by focusing on related topics such as multiscale modelling $[3,28,43]$, complexity in biology [9], systems and integrative biology [19,22,23]. Reviews dedicated

\footnotetext{
${ }^{*}$ Corresponding author. E-mail: angelique.stephanou@imag.fr
} 
to some specific applications of hybrid models can also be found, for example in biological networks modelling [12], in cancer modelling [10,21,30,40], and more specifically tumour growth [24, 29], tumour immunology [41], brain cancer [42] or angiogenesis [16]. Many special issues or reviews are also dedicated to the more specific field of hybrid systems $[2,17,31,39]$ which can be considered as a subclass of hybrid models mainly (but not only) related to the field of automatic control.

In this new review we propose a larger view on hybrid modelling in biology. We will not focus here on one particular technique or application field. We will rather propose a classification for the different types of hybrid models. We identified three main types of modelling approach that relates to both the nature and the level of coupling between the formalisms used and that qualify the models as hybrid. But before presenting this classification (section 4), we first start this review by a brief survey on the evolution of the hybrid modelling approaches from the first mention of the term "hybrid" in a context of modelling (section 2). We will then analyse the current landscape of this modelling approach based on a cloud of words generated from the overall available literature in the field (section 3).

\section{Evolution of hybrid modelling}

To illustrate the evolution of hybrid approaches in biomedicine, we used pubmed database as search engine. The key word hybrid, taken alone, produces more than 130000 references to date (August 2014). The combinations with key words model and system, taken separately, shows that the associated number of references evolves in different ways. Figure 1 shows a graphic of these evolutions from 1960 to 2013. Hybrid system starts to be significantly cited in the mid-seventies, with a threshold of 50 references attained, whereas hybrid model only reaches this threshold about 7 years later. The two evolve steadily and in parallel until the early nineties. In 1994 a dramatic increase of the number of papers related to hybrid systems is observed and intensifies to reach a pic of 1613 papers ten years later. A probable explanation for this sudden rise of hybrid system is the correlation with the development of electronics, processors and computing capabilities. In the other hand, the number of references related to hybrid model increases more steadily since hybrid model are not directly related to computing. However, we note that the progression of the last few years seem to mark the beginning of an important expanding phase, but which remains this time, difficult to correlate to any clear external factor. The increased cross-fertilization between disciplines including mathematics, computing, automatics, biology and medicine is the most logical explanation for this on-going expansion.

The association of the words hybrid with cancer, which has always been a very active field of research and which has also very early stimulated computational and mathematical modelling, shows a linear increase from the early seventies. It initially produces more references than hybrid system and hybrid model. Those two curves are eventually catching up in 1995 and 2005 respectively. Although, in this context of cancer, hybrid mainly refers to experimental models using chimera or to a combination of imaging techniques (MRI, PET, etc.) or therapeutic means (surgery, radiotherapy, chemotherapy, etc.)

Finally, if we further narrow the field to hybrid model in connection with either computational or mathematical approaches, the amount of papers only starts to be significant (i.e. above the threshold of 50 papers produced per year) in 2004. The increase has been steady over the last ten years and we are currently in the expanding phase. Almost all the references cited in this paper can be found in pubmed database using this key words sequence.

\section{The current landscape}

To get a picture of the landscape of hybrid modelling related to biology and medicine, we generated the cloud of words from the titles and abstracts of the 147 papers coming up in pubmed, in August 2014, with key words sequence: ["hybrid model" and (computational or mathematical)]. The word model 


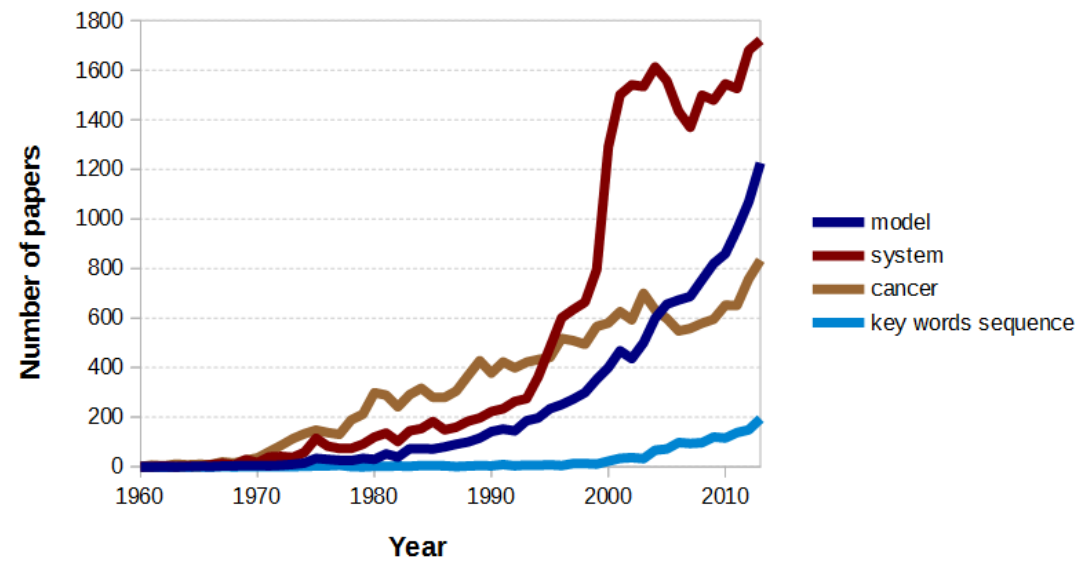

FIGURE 1. Number of papers with key words [hybrid and model], [hybrid and system], [hybrid and cancer], [hybrid model and (computational or mathematical)].

which is the most cited word has been removed and only the 100 most cited words are represented to make this cloud easier to read (Fig. 2).

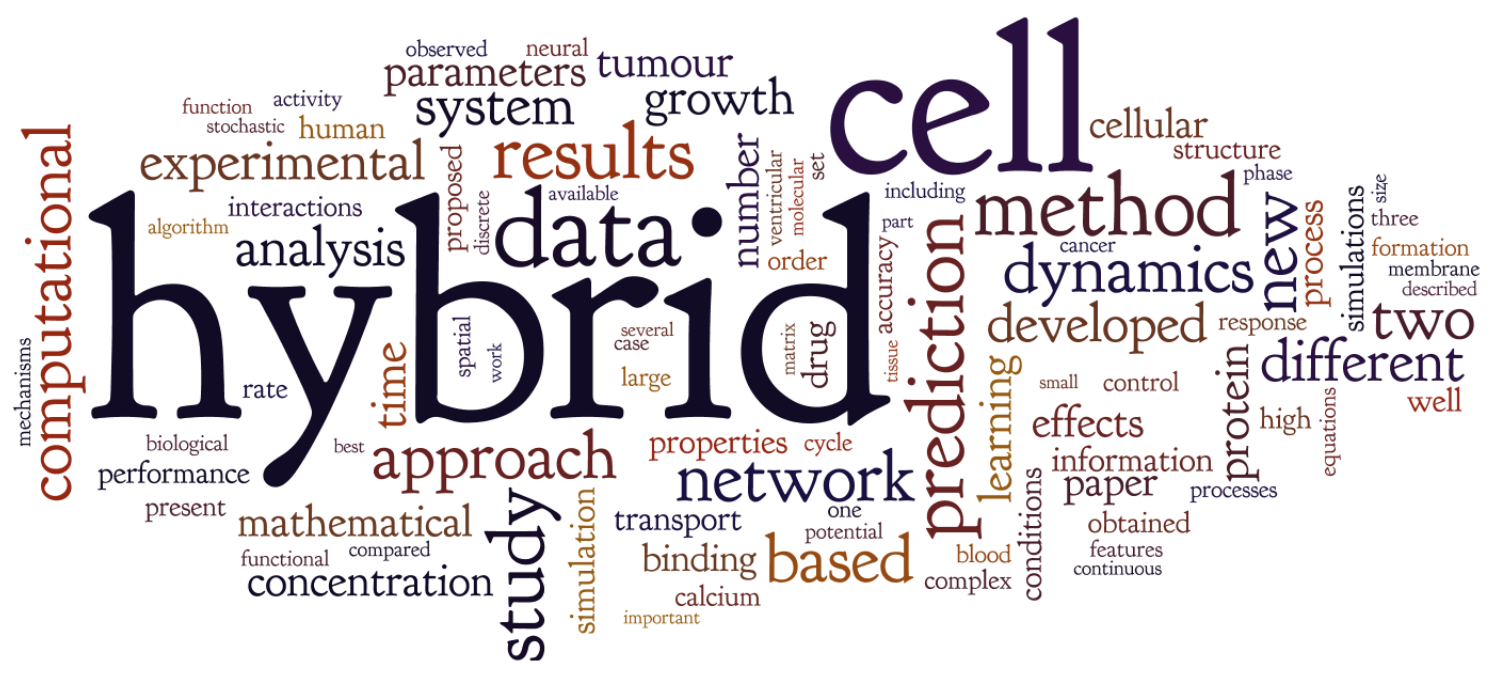

Figure 2. Cloud of words related to hybrid model (the word model has been removed).

Cell is the most cited word which is not in the key words sequence, most of the hybrid models being cell-based. We however note that cell can also refer to a grid point of a cellular automata and not exclusively to a biological cell. Data is the third most cited word. This can be explained by the fact that hybrid models mostly intend to integrate and reproduce experimental data, which also certainly explains why experimental is in the top recurring words. The function of hybrid model to be predictive is clearly highlighted by the strong recurrence of the word prediciton. The non-negligible appearance of learning might highlight that one other important goal of hybrid models is to contribute in the acquisition of new and until then inaccessible knowledge, hence the related high frequency of new. Although hybrid 
approaches are not new any more, the methods and approaches keep on developing and can be qualified as new, as well as the fields of applications that keep widening. Network is also a recurring word, but since it can be linked to many different contexts (biochemical network, gene network, interaction network, etc.) it is difficult to assess its real importance. System comes up, but its importance is relatively small since we deliberately choose to select "hybrid model" rather than "hybrid system" which is almost exclusively related to control theory.

The same frequencies of citation are observed for frequently associated words such as [discrete and continuous], [tumour (pooled with its other spelling tumor) and growth] or for words often found in a same context. For example [method, study, approach] or [simulation, dynamics, parameters, analysis, algorithm] in the context of model analysis, [performance, accuracy, control] in the context of model assessment, [protein, binding, process, transport, drug, interactions, formation] in the context or biomolecular dynamics, [human, calcium, neural, blood, membrane, cycle, cancer, ventricular, molecular, matrix, tissue] in the context of biological applications.

Interestingly, but also unsurprisingly computational largely dominates over mathematical. In the other hand, multiscale (pooled with its other spelling multi-scale) is not among the 100 most cited words although lots of hybrid models are multiscale.

Such chart is enlightening in the sense that it provides a global view of the hybrid modelling context. It would certainly be interesting to regenerate it over the years to monitor the evolution of the tendencies in this theoretical modelling field.

\section{The three types of hybrid models}

Hybrid models can be basically classified into three different types:

- independent or decoupled models,

- adjacent or coupled models,

- intricate or entangled models.

A parallel could be made between the concepts of hybridicity and disciplinarity, since decoupled models are more often associated to pluridisciplinarity, coupled models to interdisciplinarity and entangled models (with their most recent developments) to transdisciplinarity.

Case 1. Independent or decoupled models

Hybrid models of this type consists of at least two models that employ different formalisms, one discrete and one continuous for example. Those models can either describe the same phenomenon in two different ways, or two different aspects of a same phenomenon. The input and output variables in the two models are, in that case, independent and the two models can be used independently, however their synergy gives an added value to the overall hybrid approach (Fig.3, case 1).

\section{Case 2. Adjacent or coupled models}

This is the most usual and frequent type of hybrid model. In that case, two or more models, with at least two of them of a different nature (continuous/discrete, stochastic/deterministic, etc) are coupled through one or several variables (Fig.3, case 2). For example one variable is evaluated from a first continuous model and this output is used in a second discrete model. The coupling is, in most of the cases, reciprocal with a bidirectional exchange of variables (inputs and outputs).

Case 3. Intricate or entangled models

The last case is more subtle since the models involved cannot be differentiated. The model is in fact unique but its modelling potential is changing depending on the context under the form of limits, 
thresholds, switches (Hill's function) or peaks (Dirac's function) (Fig.3, case 3). These models are by essence more mathematical than computational and are more amenable to theoretical analysis (stability analysis, bifurcation, phase transition, asymptotic behaviour, etc.)

Case 1

Models are 'independent ' or de/uncoupled

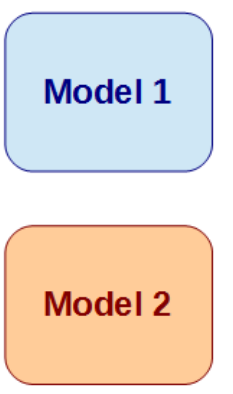

Case 2

Models are 'adjacent ' or coupled

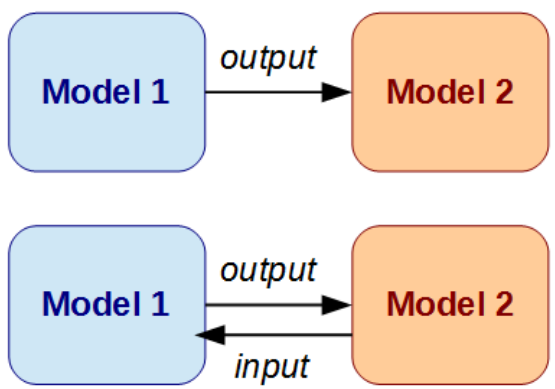

Case 3

Models are 'intricated '

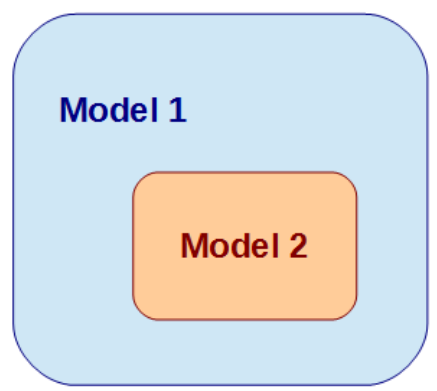

Figure 3. The three different types of hybrid models.

In the following subsections, we present some representative examples for each case.

\subsection{Decoupled models}

When the problem at hand can be divided into different parts, it is possible to model these parts independently. The modelling approaches used for each of these parts can be different by nature (continuous or discrete, stochastic or deterministic, phenomenological or physical, etc), hence the hybrid nature of the approach. In that particular case the adjective hybrid refers more to the overall approach than to the models, since the models themselves are not hybrid in most cases.

Biological networks provide a good example for this case since they possess the property of compositionality [12]. This refers to the fact that these systems can be deciphered by breaking them down into smaller and simpler pieces with well defined boundaries. The sub-systems can thus be handled individually, for example a signal transduction pathway which performs a specific (well defined) task and which involves a finite (although large) number of proteins.

Another example of hybrid approach based on independent models is to use different models to describe the same phenomena but with different levels of abstraction. Typically deterministic continuous modelling with ODEs provides a global (averaged) solution. The model can be analysed so as to evaluate its qualitative behaviour and the nature of the solutions depending on the conditions (i.e. the parameters). In the other hand, probabilistic approaches, that can typically use Markov's models, are much closer to reality and give more details including local informations. The two approaches can be used in a complementary way [12]. The first ODEs method to qualitatively analyse the model behaviour and to narrow the range of parameters for a given behaviour and the Markov's method to get more insights on the underlying key phenomena (local events, etc).

Hybrid approaches involving decoupled model can be helpful to compare some models describing the same phenomena but based on different hypotheses so as to identify the best model, that is the most accurate or the most efficient computationally. A typical example of this approach is given in the 
paper by Byrne and Drasdo (2009)[6] where the authors compare agent-based models with continuum models to describe the growth of in vitro cell aggregates. Different scenarios are tested corresponding to different cell phenotypes (either adhesive or migratory). Both models predict the same qualitative growth dynamics. They then more specifically used a cellular automaton and showed that on large scales it can be approximated by a stochastic form of the Fisher-Kolmogorov-Petrovskii-Piskounov (FKPP)-equation.

The paper by Osborne et al. (2010) [24] compares two cell-based discrete models (a cell-centre and a cell-vertex model) with a continuum model to describe the proliferation and movement of cells involved in the renewal of the intestinal crypt whose deregulation leads to colorectal cancer. The simulations show that the model exhibit similar behaviour for many critical phenomena but with however model-specific differences. The underlying question of this study was first to determine the best suited discrete approach for that particular tumour growth application, that is the approach that allow to better integrate the experimental knowledge and second, how the discrete approach can be generalised into a continuum.

\subsection{Coupled models}

The most standard form of hybrid models is to couple different models through input/output variables. A typical application for this type of hybrid model is to treat multi-scale problems [5] where different models are used to describe events occurring at different scales and the variables are passed from one scale to another. In many biological phenomena, the cell is the hub (mesoscopic scale) on which models for events occurring at the other smaller and bigger scales are plugged. Models at larger (macroscopic) scales usually describe the physical and/or chemical cell environment, and models at smaller (microscopic) scales describe the intracellular molecular dynamics from simple proteins interactions, to complex signalling cascades or metabolic circuits, down to genes expression and regulation (Fig. 4).

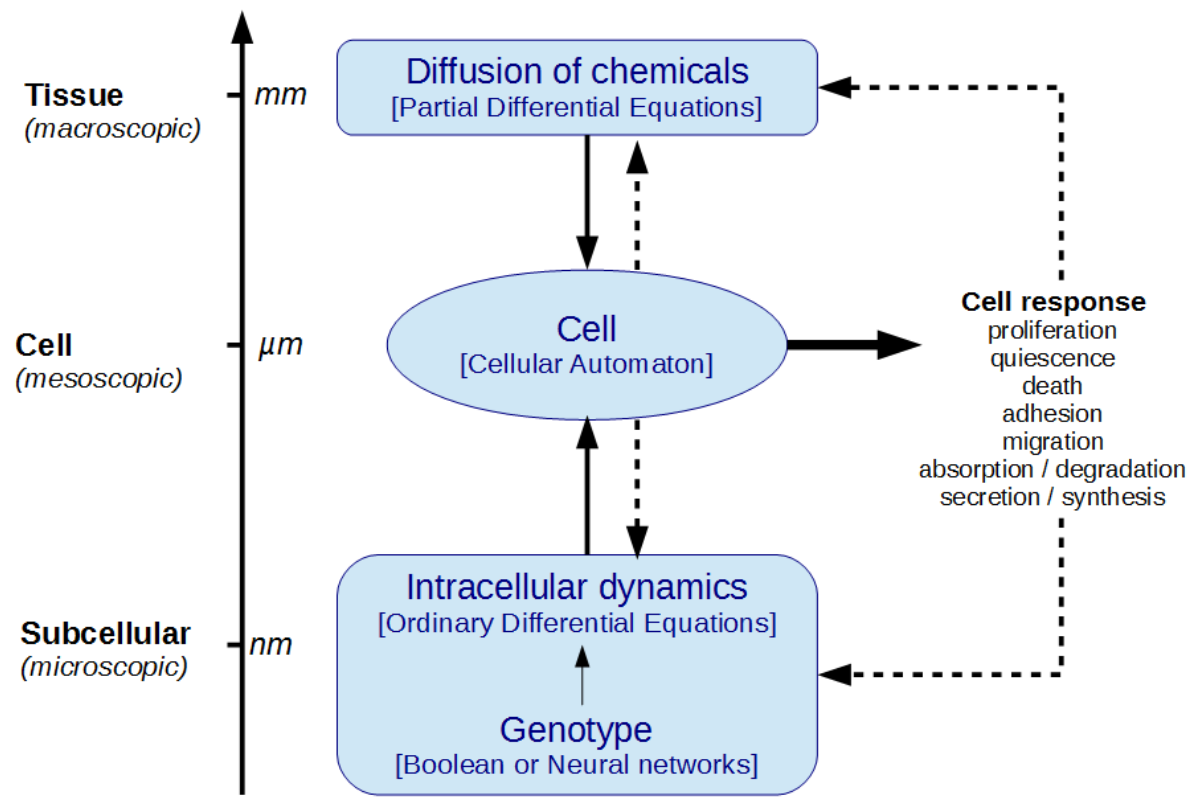

FiguRE 4. Typical structure of a cell-centred hybrid model. 


\section{Cell description with cellular automata or agent-based models}

The cell is usually represented as a discrete entity whose behaviour is determined by a set of deterministic or stochastic rules implemented in an individual-based model such as a cellular automata or an agent-based model. The difference between these two types of models lies in the representation of the cell. A cellular automaton (CA) is build as a grid (i.e. a matrix) and the cell is associated to a state (i.e. a value) of a grid point. Typically, if the grid point is occupied by a cell, the related state is 1 and 0 if there is no cell. The different types of cells are taken into account using a range of different values, for example to distinguish tumour cells from normal cells. The different cell states can also be coded in that way, for example to differentiate quiescent and proliferative cells, or any other cell status. The migration of a cell in a CA consists in propagating a state from one grid point to an adjacent one. Agent-based models in the other hand make a clear distinction between a cell (called agent) and its environment. The agent evolves autonomously and is not necessarily linked to a grid (lattice-free model). This gives more flexibility to describe a biological cell which has its own identity. ABM rely on the interactions between agents and with the environment. ABM are usually the best choice to model and simulate complex systems that cannot be globally simulated but where local rules are well known [43].

\section{Cell-plugged models}

The cell senses various signals from its environment. Its ability to process these external chemical or mechanical signals depends on the intracellular machinery which involves enzymatic and metabolic reactions. Those are classically described by mean of ordinary differential equations (ODEs) since only temporal aspects usually matters in the confined intracellular space. Boolean network are also widely used to model the regulation networks of proteins. Then depending on its intrinsic characteristics (level of differentiation, i.e. cell type) and internal state (level of protein expression, e.g. cyclins concentrations) the cells can produce a repertoire of responses which includes proliferation, differentiation, quiescence, death and migration. The cells can also produce diffusive molecules (e.g. growth factors, chemoattrants), that contribute to the changing properties of its environment. Molecular signals or chemical fields (whatever their origin: from the cell or from an external input such as a drug) are modelled as reaction-diffusion systems of partial differential equations (PDEs) (Fig. 4). Other properties of the cell environment, such as structural (homogeneity versus heterogeneity), topological (isotropy versus anisotropy) or mechanical (viscoelasticity, rigidity) properties can be modelled with either continuous or discrete models.

Coupled hybrid models are extremely vast and cover a wide spectrum of applications. Models corresponding to the framework presented in figure 4 can be found in a variety of biological applications. As a representative example, we take the review paper by Powathil and al., (2015) [26] which concerns the presentation of models developed in the context of cancer. The aims of those models is to evaluate the impact of therapies and help in therapy planning. The models need to be accurately predictive and this requires a multiscale integration of the key mechanisms responsible for tumour growth and spread from genes to tissues. At the intracellular scale, the model describes the cell cycle dynamics which is crucial to accurately describe cell proliferation. This cell cycle model involves the interactions of five proteins (including cyclins) that contributes to the cell mass evolution. The model thus consists of a system of six coupled ODEs [25]. The extracellular scale takes into account the oxygen and drug dynamics by means of PDEs. Radiation effects are also integrated using an exponential functions that depends on the level of oxygen. Intracellular and extracellular models are plugged to two different models for the discrete cells: a cellular Potts model (which is an extended cellular automaton) and a classic cellular automaton. The results obtained with these two cell models are similar and comparable which makes this double approach helpful to confirm the required accuracy of the model itself.

The cell-based multiscale framework (Fig. 4) has been used in a very similar way by Fischer et al. (2012) [11] to investigate the role of the spatial structure of erythroblastic islands in erythropoiesis. The 
hybrid multiscale model integrates an intracellular regulatory network of protein to some extracellular fields of chemicals (including growth factors) which influence together the fate of the cell represented by an off-lattice ABM. The intracellular protein competition is limited to two key proteins (Erk and Fas) and consists of two ODEs. PDEs are used to represent the diffusion of chemicals in the medium. These two continuous peripheral models determine the cell state and environmental state respectively and both states contribute in the cell fate which can either be self-renewal, differentiation or apoptotic death. This model thus provides for the first time a mean to assess the role of erythroblastic islands and of macrophages on cell proliferation and differentiation that rule erythropoiesis.

The paper by Stéphanou et al. (2015) [33] is an illustration of a bi-directional coupling with the structure as in figure 3, case 2 (lower graph). It is a hybrid discrete-continuous model which describes the individual migration of contracting cells on a an elastic matrix of fibres in the context of tumour-induced angiogenesis. The matrix is represented by a continuum whereas the cells are described as discrete elements. The tip cells forming the vascular sprouts are polarized and able to generate an oriented traction force on the matrix thanks to their discrete representation. Moreover, the cells secrete proteases as they migrate, that degrade the matrix. Proteases dynamics is modelled with a continuous diffusion equation where the source terms are the cells located at different (and sometime distant) places in the matrix. These two combined mechanical and chemical events modify the matrix topology, through oriented deformations, and property since the elasticity depends on the fibre density. If the fibres are degraded then the elasticity decreases. These local changes in the matrix reciprocally alter the migration pattern of the cells in terms of direction and velocity and this influences the resulting structure of the vasculature.

Another example of direct bi-directional coupling (Fig. 3, case 2, lower graph) is given with a model for thrombus growth. For this, a hybrid approach based on the coupling between a dissipative particle dynamics (DPD) model and PDEs was proposed [37,38]. DPD is used to model blood plasma and platelets where platelets are represented by small soft spheres which can interact with fluid particles and adhere with each other. They can aggregate in the flow or in the clot and can also detach if the force pulling them away is greater than a certain limit. PDEs are used to describe concentrations of biochemical substances in plasma, such as fibrin and thrombin in order to model the blood coagulation process. The growth of the clot, its shape and its structure depend on the blood flow intensity and reciprocally, the clot as is it growing, creates an obstacle that alters the flow.

\subsection{Intricate models}

Intricate, as we define it, means that one formalism to describe a spatially or temporally isolated event, is integrated in a general non-ambiguous formalism such as a set of mathematical equations. Those models essentially refer to mathematical modelling of non-linear dynamical systems or hybrid dynamical systems with discontinuities. We refer the interested reader to the enlightening introduction of this type of models by Aihara and Suzuki (2010) [1]. Semi-discrete approaches, used to describe systems that undergo continuous dynamics most of the time but which experience discrete events sometime, also correspond to that type of hybrid models [20]. We note that intricate models often correspond to non-spatial hybrid models where the discrete elements of the model do not need to be spatially represented. This is why the discrete aspects almost exclusively relate to time variables.

Intricate models are perhaps the most difficult (or complex) to define and to solve. The reason is that the formalism chosen is fundamentally and exclusively mathematical which is a strong constraint. The two others decoupled and coupled cases are more pragmatic in the sense that no $a$ priori constraint on the formalism is imposed and the most pertinent and helpful is simply chosen. Although the definition of such models (i.e. its transcription into equations) is more tricky, in the other hand they can be analysed and offer a better control which can be a huge added value in many cases. 
The key aspects of these models is that they integrate in a mathematical formalism some particular features of the systems such as transition or activation phenomena which create discontinuities. The inherent non-linear dynamics of the biological systems can thus appear under the form of Dirac delta function or Heaviside step function to account for threshold-triggered activation or switches between two (or more) states. It can also appear through a mixed use of continuous and discrete variables or from the use of any type of functions containing a singularity.

The catastrophe theory, formulated in the seventies by René Thom [36], is based on the latter model type containing singular functions. The models derived from this theory can thus be classified as intricate hybrid models. This theory, which is derived from the bifurcation theory, aims at studying how the parameters influence the solutions and more specifically how small changes in the parameters can lead to sharp transitions in the system behaviour. The transitions that can be observed through these systems are however limited to seven catastrophes (or topologies) which limits the types of phenomena that can be described. This theory has been applied in many different fields including biology where it was initially used by Thom to describe cell differentiation and morphogenesis. Its relevance to treat biological issues was also promoted by Zeeman [44]. However, its used was not developed further, probably due to the complexity of the formalism and the limited types of behaviours that can be generated.

A first simple illustration of intricate models can be found in the paper by Cattani and Ciancio [7] which deals with a hybrid model to study the tumour-immune system competition. The model is based on the predator-prey model of Lotka-Volterra, which is a simple non-linear system of differential equations. The model is modified so as to integrate a time-dependent stochastic parameter generated by the interaction between the tumour cells and the immune systems. This parameter stands for the cell activity which rules the cell organization depending on an information process. In that case, a hidinglearning information process can be identified, where the hiding property is associated to the uncontrolled proliferating tumour cell population $(x)$ and the learning property to the higher destructive ability of the immune cell population $(y)$. More precisely destruction is enhanced when proliferation is high so the system is adapting to its own dynamics. This is implemented through a probability function of the overall distribution that is used to modulate the activity parameter $\mu$. The related hybrid model is thus given by:

$$
\begin{aligned}
& \frac{d x}{d t}=a x-\mu(t) b x y \quad \text { with } \quad \mu(t)=1-e^{-4 \eta t} / \sqrt{2 \pi} \\
& \frac{d y}{d t}=c x y-d y
\end{aligned}
$$

where $a, b, c$ and $d$ are constants. Details on the derivation of the activity function $\mu(t)$ is given in [7]. This hybrid model produces more realistic solutions for the Lotka-Volterra system.

Intricated hybrid models can be very useful to model and investigate therapy planning since therapy corresponds to a ponctual or a repeated action through therapeutic cycles that interferes with the dynamical evolution of the disease. In the case of cancer for example, different models are required to describe tumour growth without treatment and tumour growth with treatment, since the growth dynamics is different in these two cases. To avoid this separation of models and to be able to describe the effects of treatments on a long period of time, hybrid models using switches have been developed. The model by Tanaka et al. (2010) [35] integrates in this way the alternation of on and off treatment phases on a continuous model of tumour growth dynamics involving different cell types. The model gives insights to specifically treat prostate cancer. In a similar way, Li et al. (2012) [18] proposed a hybrid model where a tumour growth model was adapted to integrate the effects of different stages of treatment. This hybrid model is given by: 


$$
\frac{d w}{d t}=\beta_{1} w s^{-}(w, \theta)+\beta_{0} s^{+}(w, \theta) \text { with } s^{+}(w, \theta)= \begin{cases}0 & w<\theta \\ 1 & w \geq \theta\end{cases}
$$

where $w$ represents the tumour weight, $\beta_{1}$ and $\beta_{0}$ are parameters characterizing the exponential and linear tumour growth rates which correspond respectively to the untreated and treated phases. $s^{+}($.$) is$ the unit step function using $\theta$ as a threshold to switch between the two phases. $s^{-}=1-s^{+}$. Simulation results produce a good match with $\mathrm{PK} / \mathrm{PD}$ data [18].

The hybrid cell cycle model of Singhania et al. (2011) [32] is another good example of intricate case. The model describes the mammalian cell cycle regulation and consists of a system of coupled ODEs to follow the evolution of the concentration levels of cyclins. The model integrates boolean variables to represent the state activity (on or off) of the regulatory proteins that control cyclin synthesis and degradation. The discrete variables which determine the rates of synthesis and degradation of the cyclins are updated through a fixed sequence of predetermined states. Reciprocally the cyclin levels determine the amount of time spent in the boolean states. The aim of this model was to introduce in a classical boolean switching network some continuous variables for cell size, cell age and cyclin levels that can be compared on a quantitative basis with experimental measurements. This hybrid approach is useful for modelling macromolecular regulatory networks in general.

Intricate models can thus be generalized so as to be used for different applications or in different contexts. The review by Singh and Hespanha (2010) [31] shows some generalized approaches to model biochemical processes. For example Galpin et al. (2008) [13] developed a modelling framework based on process algebra to describe the behaviour of hybrid biological systems. The framework allows the modelling of influences (i.e. flows) on continuous variables. The different processes that lead to changes in concentration of a species are typically production, degradation, transformation and transportation. Each of these are integrated as subcomponents which can be composed to model any system. This modelling framework has been applied to model the well-know Repressilator which is a synthetic genetic regulatory network based on three genes where the expression of one affects negatively the expression of one other. The negative feedback loop leads to oscillatory changes in protein concentrations that this new approach enables to catch.

\section{Conclusion}

In this review we proposed a classification for the hybrid models based on the level of coupling between different formalisms. We thus identified three different class of models: decoupled, coupled and intricate. The decoupled case is more a hybrid approach than a hybrid model. It is mostly used to compare the performance of different models in a range of situations. It is also useful when an overall problem can be decomposed in independent subsytems. The coupled approach is the most widely used. It is especially useful to model multiscale processes and fully connected complex systems. It is perhaps the most pragmatic and flexible approach since models can easily be constructed and connected. However the major limitation of this approach is the difficulty to analyse those models which are too heterogeneous for that exercise. In the other hand, intricate model can be analysed, however they remain limited to a reduced repertoire of applications which mainly concern the modelling of biochemical networks or other non-spatially dependent problems (e.g. therapy planning or cell cycle modelling). This class of hybrid models is strongly related to the field of hybrid systems which derived from automatics and control theory.

Considering the evolution of hybrid modelling over the last ten years, those models are about to become ubiquitous in biology and in medicine. The increased access to data in these fields always requires more integrated models and more sophisticated hybrid techniques will keep developing. The urgent need is to develop approaches that can be both true to the experimental context (i.e. true to 
the data) but also that can allow some control on the model where the analysis of its behaviour should be made possible. Currently the only way to analyse computationally based hybrid models is to run a huge amount of simulations in order to assess the sensitivity and robustness of the parameters through statistical analyses. This constrains the modeller to make numerical experimentations [14] and analyse the results in the same way as an experimental biologist. There is today a growing concern for the development of new modelling techniques which better integrate the different scales [8], which provide an increased control on the system and which give some new mean to analyse the models and results [15]. Examples of such new techniques can be found in a recent special issue on hybrid models in cell biology, published in Mathematical Modelling and Natural Phenomena (Volume 10, Issue 1, January 2015).

Acknowledgements. The second author is supported by the grant of Russian Science Foundation, project no. 15-11-00029.

\section{References}

[1] K. Aihara, H. Suzuki. Theory of hybrid dynamical systems and its applications to biological and medical systems. Phil. Trans. R. Soc. A (2010), 368, 4893-4914.

[2] P.J. Antsaklis. Special issue on hybrid system: theory and applications. A brief introduction to the theory and applications of hybrid systems. Proceedings of the IEEE (2000), 88(7), 879-887.

[3] S. Bernard. How to build a multiscale model in biology. Acta biotheor. (2013), 61, 291-303.

[4] N. Bessonov, F. Crauste, S. Fischer, P. Kurbatova, V. Volpert. Application of hybrid models to blood cell production in the bone marrow. Math. Mod. Nat. Phenom. (2011), 6(7), 2-12.

[5] B.S. Brooks, S.L. Waters. Mathematical challenges in integrative physiology. J.Math. Biol. (2008), 56, 893-896.

[6] H. Byrne and D. Drasdo. Individual-based and continuum models of growing cell populations: a comparison. J. Math. Biol. (2009), 58, 657-687.

[7] C. Cattani, A. Ciancio. Separable transition density in the hybrid model for tumor-immune system competition. Comp. Math. Meth. Med. (2012), 610124.

[8] A. Colombi, M. Scianna, L. Preziosi. A measure-theoretic model for collective cell migration and aggregation. Math. Mod. Nat. Phenom. (2015), 10(1), 4.

[9] P.V. Coveney, P.W. Fowler. Modelling biological complexity: a physical scientist's perspective. J. R. Soc. Interface (2005), 2, 267-280.

[10] T.S. Deisboeck, Z. Wang, P. Macklin, V. Cristini. Multiscale cancer modeling. Annu. Rev. Biomed. Eng. (2011), 13, 127-155.

[11] S. Fischer, P. Kurbatova, N. Bessonov, O. Gandrillon, V. Volpert, F. Crauste. Modeling erythroblastic islands: Using a hybrid model to assess the function of central macrophage. J. theor. Biol. (2012), 298, 92-106.

[12] J. Fisher, N. Piterman. The executable pathway to biological networks. Briefings in functional genomics (2010), 9(1), 79-92.

[13] V. Galpin, J. Hillston, L. Bortolussi. HYPE applied to the modelling of hybrid biological systems. Electronic Notes in Theoretical Computer Science (2008), 218, 33-51.

[14] N. Glade, A. Stéphanou. Le vivant discret et continu: modes de représentation en biologie théorique. Editions Matériologiques, Paris, 2013.

[15] P. Guerrero, T. Alarcón. Stochastic multiscale models of cell population dynamics: asymptotic and numerical methods. Math. Mod. Nat. Phenom. (2015), 10(1).

[16] T.A.M Heck, M.M. Vaeyens, H. Van Oosterwyck. Computational models of sprouting angiogenesis and cell migration: towards multiscale mechanochemical models of angiogenesis. Math. Mod. Nat. Phenom. (2015), 10(1).

[17] W.P.M.H. Heemels, B. De Schutter, J. Lunze, M. Lazar. Stability analysis and controller synthesis for hybrid dynamical systems. Phil. Trans. R. Soc. A (2010), 368, 4937-4960.

[18] X. Li, L. Qian, M.L. Bittner, E.R. Dougherty. A systems biology approach in therapeutic response study for different dosing regimens - a modeling study of drug effects on tumor growth using hybrid systems. Cancer Informatics (2012), $11,41-60$.

[19] D. Machado, R.S. Costa, M. Rocha, E.C. Ferreira, B. Tidor, I. Rocha. Modeling formalisms in systems biology. AMB Express (2011), 1, 45.

[20] L. Mailleret, V. Lemesle. A note on semi-discrete modelling in the life sciences. Phil. Trans. R. Soc. A (2009), 367, 4779-4799.

[21] A. Masoudi-Nejad, E. Wang. Cancer modeling and network biology: accelerating toward personalized medicine. Seminars in Cancer Biology (2015), 30, 1-3.

[22] B. Mishra. Intelligently deciphering unintelligible designs: algorithmic algebraic model checking in systems biology. J. R. Soc. Interface (2009), 6, 575-597.

[23] T. Nomura. Toward integration of biological and physiological functions at multiple levels. Frontiers in Physiology (2010), 1, 164. 
[24] J.M. Osborne, A. Walter, S.K. Kershaw, G.R. Mirams, A.G. Fletcher, P. Pathmanathan, D. Gavaghan, O.E. Jensen, P.K. Maini, H.M. Byrne. A hybrid approach to multi-scale modelling of cancer. Phil. Trans. R. Soc. A (2010), 368, 5013-5028.

[25] G.G. Powathil, K.E. Gordon, L.A. Hill, M.A. Chaplain. Modelling the effects of cell-cycle heterogeneity on the response of a solid tumour to chemotherapy: biological insights from a hybrid multiscale cellular automaton model. J. theor. Biol. (2012), 308, 1-9.

[26] G.G. Powathil, M. Swat, M.A.J. Chaplain. Systems oncology: Towards patient-specific treatment regimes informed by multiscale modelling. Seminars in Cancer Biology (2015), 30, 13-20.

[27] L. Preziosi. Hybrid and multiscale modelling. J. Math. Biol. (2006), 53, 977-978.

[28] Z. Qu, A. Garfinkel, J.N. Weiss, M. Nivala. Multi-scale modeling in biology: How to bridge the gaps beween scales? Prog. Biophys. Mol. Biol. (2011), 107, 21-31.

[29] K.A. Rejniak, A.R.A. Anderson. Hybrid models if tumor growth. Wiley Interdiscipl. Rev. Syst. Biol. Med. (2011), 3(1), 115-125.

[30] S. Sanga, H.B. Frieboes, X. Zheng, R. Gatenby, E.L. Bearer, V. Cristini. Predictive oncology: multidisciplinary, multiscale in-silico modeling linking phenotype, morphology and growth. Neuroimage (2007), 37(1), S120-S134.

[31] A. Singh, J.P. Hespanha. Stochastic hybrid systems for studying biochemical processes. Phil. Trans. R. Soc. A (2010), 368, 4995-5011.

[32] R. Singhania, R.M. Sramkoski, J.W. Jacobberger, J.J. Tyson. A hybrid model of mammalian cell cycle regulation. PLoS Comput. Biol. (2011), 7(2), e1001077.

[33] A. Stéphanou, S. Le Floc'h, A. Chauvière. A hybrid model to test the importance of mechanical cues driving cell migration in angiogenesis. Math. Mod. Nat. Phenom. (2015), 10(1).

[34] A. Stéphanou, V. Volpert. Hybrid modelling in cell biology. Math. Mod. Nat. Phenom. (2015), 10(1), 1-3.

[35] G. Tanaka, Y. Hirata, S.L. Goldenberg, N. Bruchovsky, K. Aihara. Mathematical modelling of prostate cancer growth and its application to hormone therapy. Phil. Trans. R. Soc. A (2010), 368, 5029-5044.

[36] R. Thom. Stabilité structurelle et morphogenèse. Interédition, Paris, 1977.

[37] A. Tosenberger, F. Ataullakhanov, N. Bessonov, M. Panteleev, A. Tokarev, V. Volpert. Modelling of thrombus growth in flow with a DPD-PDE-method. J. theor. Biol. (2013), 337, 30-41.

[38] A. Tosenberger, N. Bessonov, V. Volpert. Influence of blood coagulation on clot formation in flow by a hybrid model. Math. Mod. Nat. Phenom. (2015), 10(1), 36.

[39] D. Vries, P.J.T. Verheijen, A.J. den Dekker. Hybrid system modeling and identification of cell biology systems: perpective and challenges. Symposium on system identification (2009), 227-232.

[40] Z. Wang, J.D. Butner, R. Kerketta, V. Cristini. Simulating cancer growth with multiscale agent-based modeling. Seminars in cancer biology (2015), 30.

[41] A.L. Woelke, M.S. Murgueitio, R. Preissner. Theoretical modeling techniques and their impact on tumor immunology. Clinical and Developmental Immunology (2010), 271794.

[42] L. Zhang, L.L. Chen, T.S. Deisboeck. Multi-scale, multi-resolution brain cancer modeling. Math. Comput. Simul. (2009), 79(7), 2021-2035.

[43] L. Zhang, Z. Wang, J.A. Sagotsky, T.S. Deisboeck. Multiscale agent-based cancer modeling. J. Math. Biol. (2009), 58, $545-559$.

[44] E.C. Zeeman. Levels of structure in catastrophe theory illustrated by applications in the social and biological sciences, Proceedings of the International Congress of Mathematicians (Vancouver, 1974) 2:533-546, Canad. Math. Congress, Montreal, 1975. 